\title{
An Improved Sharp Interface Method for Viscoelastic and Viscous Two-Phase Flows
}

\author{
P.A. Stewart • N. Lay $\cdot$ M. Sussman • M. Ohta
}

Received: 20 February 2007 / Revised: 9 October 2007 / Accepted: 5 November 2007

(C) Springer Science+Business Media, LLC 2007

\begin{abstract}
We introduce a robust method for computing viscous and viscoelastic two-phase bubble and drop motions. Our method utilizes a coupled level-set and volume-of-fluid technique for updating and representing the air-water interface. Our method introduces a novel approach for treating the viscous coupling terms at the air-water interface; these improvements result in improved stability for computing two-phase bubble formation solutions. We also present an improved, "positive-preserving" discretization technique for updating the configuration tensor for viscoelastic flows, in the context of computing two-phase bubble and drop motion.
\end{abstract}

Keywords Level-set method · Volume-of-fluid method $\cdot$ Two-phase flow $\cdot$ Bubbles $\cdot$ Drops

\section{Introduction}

In [1, 2], and [3], a sharp interface method was developed for computing solutions to problems in Newtonian two-phase flows and viscoelastic two-phase flows. The interface separating the two phases in these papers was represented and updated using the coupled level set and volume-of-fluid method. The treatment of interfacial boundary conditions for our sharp interface method has the property that in the limit of zero gas density and zero gas viscosity, i.e. in the limit that the gas is treated as a zero density void, the solutions approach the solutions of the corresponding one-phase problem. The air-water interface for the one-phase problem is a free-surface in which the liquid on one side is treated as an incompressible fluid and the gas pressure on the other side of the free-surface is treated as spatially constant.

P.A. Stewart $\cdot$ N. Lay $\cdot$ M. Sussman $(\bowtie)$

Department of Mathematics, Florida State University, Tallahassee, FL 32306, USA

e-mail: sussman@math.fsu.edu

M. Ohta

Department of Applied Chemistry, Muroran Institute of Technology, 27-1, Mizumotocho, Muroran-shi, Hokkaido 050-8585, Japan

e-mail: mohta@mmm.muroran-it.ac.jp 
We remark that Kang et al. [4] used the Ghost Fluid Method [5] and techniques in [6] to treat multiphase incompressible flow, including the effects of viscosity, surface tension, and gravity using a sharp interface method. Their method takes the interfacial jump conditions into consideration, but they use an explicit formulation of viscous terms. References [13 ], and this paper utilize an implicit treatment of the viscous terms. Our treatment takes the location of the interface into consideration and uses the knowledge that the two-phase algorithm reduces to a one-phase algorithm in the limit that gas becomes a constant pressure void.

In this paper we present two improvements, in the context of our sharp interface method; (1) we present improvements for the discretization of the "coupling" terms that appear in the viscous force terms and (2) we present improved discretization techniques for updating the configuration tensor for viscoelastic flows.

\subsection{Improvements in Calculating the Viscous Force Terms}

We introduce a robust method for computing viscosity in two-phase bubble and drop motions. Sussman et al. [1] described a sharp interface method for incompressible two-phase flows. This method uses a coupled level-set and volume-of-fluid technique for updating and representing the air-water interface, and introduces a novel approach for treating the jump conditions at the air-water interface. This method yielded solutions in the zero gas density limit which were comparable in accuracy to the method in which the gas pressure was treated as spatially constant. This improvement allowed for increases in the accuracy of solutions on coarse grids.

However, the above method's matrix solver would not converge when viscous effects were dominant. The above method also was inefficient in the sense that it used a second order time discretization, even though the spatial error in discretizing the viscous force terms was only first order accurate. Li et al. [7] gave a method to decouple problematic viscous terms with a low Reynolds number. This provided a basis for Sussman and Ohta to describe improvements for calculating two-phase bubble and drop motions [3].

The new method proposed by Sussman-Ohta [3] no longer suffered from problems with large viscous terms. The decoupling of the viscous terms resulted in a diagonally dominant matrix system for the non-coupled viscous force terms; such a matrix system could be robustly solved using the conjugate gradient method or multigrid method. This new method involved a first order, backwards Euler time discretization for the non-coupled viscous terms and was comparably better than [1] when viscous effects are dominant.

However, the calculation of the viscous coefficients used for the coupling terms in [3] are not as accurate as they could be, and the method will lead to instabilities in some calculations. The analysis done in [7] only considered the temporal discretization when proving unconditional stability for their method. We show through example in this paper that the semi-implicit approach taken by [3] works well for many bubble and drop two-phase flow problems, but fails for the bubble formation problem for certain parameter regimes. Here, we shall introduce a new method to improve the evaluations of the viscous coupling-term coefficients. Our method does not lead to instabilities and is either just as accurate as the methods proposed in [1] and [3], or for some problems, measurably more accurate.

Other methods of interest include [8] and [9]. In [8], Hong et al. treats their viscous terms implicitly in a method similar to that described in [3]. However, they assume that the net result of their viscous coupling terms is zero. In [9], Rasmussen et al. also use a method similar to that described in [3]. However, their method for discretizing the viscous force terms is developed in the context of one-phase flow with variable liquid viscosity, instead 
of two-phase flow; we focus on the treatment of the viscous force terms at a two-phase interface where both density and viscosity have large jumps.

\subsection{A Positive Definite Preserving Numerical Scheme for Updating the Configuration} Tensor for Viscoelastic Two-Phase Flow

Algorithms for viscoelastic two-phase flow have been presented by Yu et al. [10], Pillapakkam and Singh [11], and Jimenez et al. [2]. Also, algorithms for viscoelastic one-phase flow have been presented by Goktekin et al. [12], Losasso et al. [13] and Irving [14]. We remark that the previous methods [10] and [11] were developed in the context of a "smoothed" interface method, instead of the sharp interface method presented here. The work by [12-14] was developed in the context of a one-phase flow algorithm in which the gas pressure was assumed spatially constant. We note that, as remarked by Losasso et al. [13], Goktekin et al. [12] ignored the fluid rotation term since Goktekin et al. solved the following simplified equation for the elastic strain tensor $\varepsilon$ :

$$
\varepsilon_{t}+\vec{u} \cdot \varepsilon=\left(\nabla \vec{u}+(\nabla \vec{u})^{T}\right) / 2-\varepsilon_{t}^{\text {Plastic }} .
$$

In this paper, we describe improvements to the work presented by Jimenez et al. [2] for the discretization of the evolution equation for the configuration tensor $A$ :

$$
\frac{\partial \mathbf{A}}{\partial t}+\mathbf{u} \cdot \nabla \mathbf{A}=\mathbf{A} \cdot(\nabla \mathbf{u})^{T}+\nabla \mathbf{u} \cdot \mathbf{A}-\frac{f(R)}{\lambda}(\mathbf{A}-\boldsymbol{I})
$$

For the FENE-CR model [15], the conformation of the polymer is specified in terms of the average of the dyadic product $\langle\mathbf{R R}\rangle$ of the dumbbell end-to-end vector $\mathbf{R}$. $c, L, \lambda$ are FENECR model parameters. The parameter $c$ is a measure of the concentration of dumbbells, $L$ is the extensibility parameter which denotes the maximum average length of $s$ polymer molecule relative to the equilibrium end-to-end dimension, $\lambda$ is a characteristic relaxation time. The FENE-CR model has a constant shear viscosity and the FENE-CR model reduces to the Oldroyd-B model as $L \rightarrow \infty$. The function $f(R)$ specifies the nonlinear spring characteristics of the viscoelastic fluid and is given by

$$
f(R)=\frac{1}{1-\operatorname{tr}(\mathbf{A}) / L^{2}} .
$$

In particular, we observe that (1.1) preserves $A$ as a positive definite matrix. The discretization of (1.1) should preserve the positive definite property of $A$ as well. In our previous work [2], we only preserved the diagonal entries of $A$ to be positive. We remark that there are many choices of schemes for discretizing (1.1), see e.g. [16, 17] and [18], but they are complicated, and ultimately one must reduce the order of accuracy of a method to first order in order to preserve positive definiteness of $A$. We instead base our discretization on the following observation that one can rewrite (1.1) as follows,

$$
A(x, t+\Delta t)=I+\left((I+\Delta t \nabla U) A(x-U \Delta t, t)(I+\Delta t \nabla U)^{T}-I\right) e^{\frac{-f(R)}{\lambda} \Delta t}+O\left(\Delta t^{2}\right) .
$$

We show that we can robustly calculate viscoelastic bubbly flows using our new method for updating the configuration tensor together with our sharp interface approach.

We remark that a similar "matrix transformation" technique as given in (1.3) was also implemented by [14]. I.e., [14] also implemented a semi-Lagrangian advection step to 
find $\varepsilon(x-U \Delta t, t)$ and then computed the similarity transformation $S \varepsilon S^{T}$ where $S=$ $\frac{1}{2}\left(\nabla U-\nabla U^{T}\right)$. The viscoelastic model in [14] was based on the corotational Maxwell model and the viscoelastic model in this paper is based on the FENE-CR model. The numerical discretization in [14] does not preserve the positive definite property of their elastic strain tensor, whereas our numerical discretization does preserve the configuration tensor, $A$, as a positive definite matrix.

\section{Governing Equations}

The governing equations for incompressible, immiscible two-phase flow was written by Chang et al. [19] as:

$$
\begin{gathered}
\rho \frac{D U}{D t}=\nabla \cdot\left(-p I+2 \mu D+\frac{\tilde{\eta}_{P}}{\lambda} f(R) A\right)+\rho g \hat{z}-\sigma \kappa(F) \nabla H, \\
\frac{\partial \mathbf{A}}{\partial t}+U \cdot \nabla \mathbf{A}=\mathbf{A} \cdot(\nabla U)^{T}+\nabla U \cdot \mathbf{A}-\frac{f(R)}{\lambda}(\mathbf{A}-\mathbf{I}), \\
\nabla \cdot U=0, \quad \frac{D F}{D t}=0, \\
\frac{D \phi}{D t}=0, \quad \mu=\mu_{L} H(\phi)+\mu_{G}(1-H(\phi)), \quad \tilde{\eta}_{P}=\eta_{P} H(\phi), \\
\rho=\rho_{L} H(\phi)+\rho_{G}(1-H(\phi)), \quad \\
H(\phi)= \begin{cases}1, & \phi \geq 0, \\
0, & \phi<0,\end{cases}
\end{gathered}
$$

$\phi$ is a level set function which is positive in liquid and negative in gas. $F$ is a volume-of-fluid function which represents the fraction of fluid that is liquid for each computational cell. Our method follows the time-splitting that was used in Sussman and Ohta [3] for decoupling the viscous terms, and also uses the previously developed CLSVOF method by Sussman and Puckett [20] by coupling the solutions of $\phi$ and $F$.

Other variables used are defined as: $U$ is the velocity field, $p$ is the pressure, $D=$ $\frac{1}{2}\left(\nabla U+\nabla U^{T}\right)$ is the rate of deformation tensor, $g$ corresponds to the acceleration due to gravity, $\mu_{L}\left(\mu_{G}\right)$ is the viscosity of liquid (gas), $\rho_{L}\left(\rho_{G}\right)$ is the density of liquid (gas), $\kappa(F)=\nabla \cdot \frac{\nabla F}{|\nabla F|}$ is the curvature, and $\sigma$ is the coefficient of surface tension. The gradient terms on the right hand side of the equation for $A$ are defined as

$$
\mathbf{A} \cdot(\nabla U)^{T}+\nabla U \cdot \mathbf{A}=\mathbf{A}_{\alpha \gamma} \frac{\partial U_{\beta}}{\partial x_{\gamma}}+\frac{\partial U_{\alpha}}{\partial x_{\gamma}} \mathbf{A}_{\gamma \beta} .
$$

\section{Viscosity: An Adaptive, Sharp Interface Treatment for the Viscous Force Terms}

We present a simple and robust adaptive method for computing the viscous forces as they appear in (2.1), 
Our algorithm follows the same strategy as proposed by Li, Renardy, and Renardy [7] and as implemented in Sussman-Ohta [3] by discretizing the coupling terms in (3.1) explicitly and the remainder of the terms implicitly. It was shown by Li et al. [7] that the following temporal discretization for the viscous forces is stable for any time step:

$$
\begin{gathered}
\rho \frac{u^{* *}-u^{*}}{\Delta t}=\nabla \cdot \mu \nabla u^{* *}+\left(\mu u_{x}^{* *}\right)_{x}+\left(\mu v_{x}^{*}\right)_{y}+\left(\mu w_{x}^{*}\right)_{z}, \\
\rho \frac{v^{* *}-v^{*}}{\Delta t}=\nabla \cdot \mu \nabla v^{* *}+\left(\mu v_{y}^{* *}\right)_{y}+\left(\mu u_{y}^{*}\right)_{x}+\left(\mu w_{y}^{*}\right)_{z}, \\
\rho \frac{w^{* *}-w^{*}}{\Delta t}=\nabla \cdot \mu \nabla w^{* *}+\left(\mu w_{z}^{* *}\right)_{z}+\left(\mu u_{z}^{*}\right)_{x}+\left(\mu v_{z}^{*}\right)_{y} .
\end{gathered}
$$

The non-coupling terms are discretized using standard finite volume techniques. For example, we approximate the terms $\nabla \cdot \mu \nabla u^{* *}+\left(\mu u_{x}^{* *}\right)_{x}$ from above as,

$$
\begin{array}{r}
\frac{2 \mu_{i+1 / 2, j, k}\left(u_{i+1, j, k}-u_{i, j, k}\right)-2 \mu_{i-1 / 2, j, k}\left(u_{i, j, k}-u_{i-1, j, k}\right)}{\Delta x^{2}} \\
+\frac{\mu_{i, j+1 / 2, k}\left(u_{i, j+1, k}-u_{i, j, k}\right)-\mu_{i, j-1 / 2, k}\left(u_{i, j, k}-u_{i, j-1, k}\right)}{\Delta y^{2}} \\
+\frac{\mu_{i, j, k+1 / 2}\left(u_{i, j, k+1}-u_{i, j, k}\right)-\mu_{i, j, k-1 / 2}\left(u_{i, j, k}-u_{i, j, k-1}\right)}{\Delta z^{2}} .
\end{array}
$$

The face centered viscosity, $\mu_{i+1 / 2, j}$, is defined "sharply" as,

$$
\mu_{i+1 / 2, j}=\frac{1}{\frac{\theta_{i+1 / 2, j}}{\mu_{L}}+\frac{1-\theta_{i+1 / 2, j}}{\mu_{G}}} .
$$

$\theta_{i+1 / 2, j}$ is the height fraction [7, 8] given by:

$$
\theta_{i+1 / 2, j}(\phi)= \begin{cases}1, & \phi_{i+1, j}, \phi_{i, j} \geq 0, \\ 0, & \phi_{i+1, j}, \phi_{i, j}<0, \\ \frac{\phi_{i+1, j}^{+}+\phi_{i, j}^{+}}{\left|\phi_{i+1, j}\right|+\left|\phi_{i, j}\right|}, & \text { otherwise. }\end{cases}
$$

The "+" superscript stands for the positive part, i.e. $a^{+} \equiv \max (a, 0)$. The combined density is also defined sharply as,

$$
H(\rho)= \begin{cases}\rho^{L}, & \phi \geq 0, \\ \rho^{G}, & \phi<0 .\end{cases}
$$

In Sussman and Ohta [3], the coupling terms were discretized in two dimensions as

$$
\begin{aligned}
\frac{\partial\left(\mu v_{x}\right)}{\partial y} \approx & \left(\mu_{i+1 / 2, j+1 / 2}\left(v_{x}\right)_{i+1 / 2, j+1 / 2}-\mu_{i+1 / 2, j-1 / 2}\left(v_{x}\right)_{i+1 / 2, j-1 / 2}\right. \\
& \left.+\mu_{i-1 / 2, j+1 / 2}\left(v_{x}\right)_{i-1 / 2, j+1 / 2}-\mu_{i-1 / 2, j-1 / 2}\left(v_{x}\right)_{i-1 / 2, j-1 / 2}\right) / 2 \Delta y \\
\frac{\partial\left(\mu u_{y}\right)}{\partial x} \approx & \left(\mu_{i+1 / 2, j+1 / 2}\left(u_{y}\right)_{i+1 / 2, j+1 / 2}-\mu_{i-1 / 2, j+1 / 2}\left(u_{y}\right)_{i-1 / 2, j+1 / 2}\right. \\
& \left.+\mu_{i+1 / 2, j-1 / 2}\left(u_{y}\right)_{i+1 / 2, j-1 / 2}-\mu_{i-1 / 2, j-1 / 2}\left(u_{y}\right)_{i-1 / 2, j-1 / 2}\right) / 2 \Delta x
\end{aligned}
$$


The viscosity at a node was given by

$$
\mu_{i+1 / 2, j+1 / 2}= \begin{cases}\mu_{L}, & \theta_{i+1 / 2, j+1 / 2}=1, \\ \mu_{G}, & \theta_{i+1 / 2, j+1 / 2}=0, \\ 0, & \mu_{G}=0 \text { and } 0<\theta_{i+1 / 2, j+1 / 2}<1, \\ \mu_{L G}, & \text { otherwise }\end{cases}
$$

where

$$
\mu_{L} G=\frac{\mu_{G} \mu_{L}}{\mu_{G} \theta_{i+1 / 2, j+1 / 2}+\mu_{L}\left(1-\theta_{i+1 / 2, j+1 / 2}\right)} .
$$

$\theta_{i+1 / 2, j+1 / 2}$ is a "node fraction" defined as,

$$
\theta_{i+1 / 2, j+1 / 2}(\varphi)= \begin{cases}1, & \phi_{i+1, j}, \phi_{i, j}, \phi_{i, j+1}, \phi_{i+1, j+1} \geq 0 \\ 0, & \phi_{i+1, j}, \phi_{i, j}, \phi_{i, j+1}, \phi_{i+1, j+1}<0 \\ \theta_{N D}, & \text { otherwise. }\end{cases}
$$

The "+" superscript stands for the positive part, i.e. $a^{+} \equiv \max (a, 0)$, and

$$
\theta_{N D}=\frac{\phi_{i+1, j}^{+}+\phi_{i, j+1}^{+}+\phi_{i, j}^{+}+\phi_{i+1, j+1}^{+}}{\left|\phi_{i+1, j}\right|+\left|\phi_{i, j+1}\right|+\left|\phi_{i, j}\right|+\left|\phi_{i+1, j+1}\right|} .
$$

The components of the parts of the deformation tensor that are handled explicitly, e.g. the coupled terms, $\left(u_{y}\right)_{i+1 / 2, j+1 / 2}$ in the equation for $v$ and $\left(v_{x}\right)_{i+1 / 2, j+1 / 2}$ in the equations for $u$, were calculated at nodes using standard central differencing, i.e.,

$$
\left(u_{y}\right)_{i+1 / 2, j+1 / 2}=\frac{u_{i+1, j+1}-u_{i+1, j}+u_{i, j+1}-u_{i, j}}{2 \Delta y} .
$$

The discretization of the viscous terms in [3] had the following properties:

1. If the gas viscosity is zero, the velocity in gas cells, $\varphi_{i, j}<0$, is never used. This enabled our two-phase method to be equivalent to the corresponding one-phase method in the limit of zero gas density and zero gas viscosity (i.e. gas treated as vacuum with uniform pressure).

2. The resulting matrix system for each velocity component is written in the following form,

$$
\alpha(x) p+\beta \nabla \cdot(A(x, y) \nabla p)=f(x, y) .
$$

Where (3.3) is solved for p. A is a diagonal matrix. Reference [3] solved (3.3) on an adaptive hierarchy of grids as also described in Sect. 5 .

In this paper, we discretize the coupling terms differently from [3]; in three dimensions, they appear as:

$$
\begin{aligned}
\left.\frac{\partial u}{\partial y}\right|_{i-1 / 2, j, k}= & \frac{\omega_{i, j+1, k}^{i, j, k}\left(u_{i, j+1, k}-u_{i, j, k}\right)+\omega_{i, j, k}^{i, j-1, k}\left(u_{i, j, k}-u_{i, j-1, k}\right)}{\Delta y\left(\omega_{i, j+1, k}^{i, j, k}+\omega_{i, j, k}^{i, j-1, k}+\omega_{i-1, j+1, k}^{i-1, j, k}+\omega_{i-1, j, k}^{i-1, j-1, k}\right)} \\
& +\frac{\omega_{i-1, j+1, k}^{i-1, j, k}\left(u_{i-1, j+1, k}-u_{i-1, j, k}\right)+\omega_{i-1, j, k}^{i-1, j-1, k}\left(u_{i-1, j, k}-u_{i-1, j-1, k}\right)}{\Delta y\left(\omega_{i, j+1, k}^{i, j, k}+\omega_{i, j, k}^{i, j-1, k}+\omega_{i-1, j+1, k}^{i-1, j, k}+\omega_{i-1, j, k}^{i-1, j-1, k}\right)},
\end{aligned}
$$




$$
\begin{aligned}
\left.\frac{\partial u}{\partial z}\right|_{i-1 / 2, j, k}= & \frac{\omega_{i, j, k+1}^{i, j, k}\left(u_{i, j, k+1}-u_{i, j, k}\right)+\omega_{i, j, k}^{i, j, k-1}\left(u_{i, j, k}-u_{i, j, k-1}\right)}{\Delta z\left(\omega_{i, j, k+1}^{i, j, k}+\omega_{i, j, k}^{i, j, k-1}+\omega_{i-1, j, k+1}^{i-1, j, k}+\omega_{i-1, j, k}^{i-1, j, k-1}\right)} \\
& +\frac{\omega_{i-1, j, k+1}^{i-1, j, k}\left(u_{i-1, j, k+1}-u_{i-1, j, k}\right)+\omega_{i-1, j, k}^{i-1, j-1}\left(u_{i-1, j, k}-u_{i-1, j, k-1}\right)}{\Delta z\left(\omega_{i, j, k+1}^{i, j, k}+\omega_{i, j, k}^{i, j, k-1}+\omega_{i-1, j, k+1}^{i-1, j, k}+\omega_{i-1, j, k}^{i-1, j, k-1}\right)},
\end{aligned}
$$

where,

$$
\begin{aligned}
\omega_{I, J, K}^{L, M, N} & =\left\{\begin{array}{ll}
1, & s \phi_{I, J, K} \geq 0 \text { and } s \phi_{L, M, N} \geq 0, \\
0, & \text { otherwise }
\end{array} \quad\right. \text { and } \\
s & = \begin{cases}1, & \phi_{i-1, j, k} \geq 0 \text { or } \phi_{i, j, k} \geq 0, \\
-1, & \text { otherwise },\end{cases}
\end{aligned}
$$

$$
\begin{aligned}
\left.\frac{\partial v}{\partial x}\right|_{i, j-1 / 2, k}= & \frac{\omega_{i+1, j, k}^{i, j, k}\left(v_{i+1, j, k}-v_{i, j, k}\right)+\omega_{i, j, k}^{i-1, j, k}\left(v_{i, j, k}-v_{i-1, j, k}\right)}{\Delta x\left(\omega_{i+1, j, k}^{i, j, k}+\omega_{i, j, k}^{i-1, j, k}+\omega_{i+1, j-1, k}^{i, j-1, k}+\omega_{i, j-1, k}^{i-1, j-1, k}\right)} \\
& +\frac{\omega_{i+1, j-1, k}^{i, j-1, k}\left(v_{i+1, j-1, k}-v_{i, j-1, k}\right)+\omega_{i, j-1, k}^{i-1, j-1, k}\left(v_{i, j-1, k}-v_{i-1, j-1, k}\right)}{\Delta x\left(\omega_{i+1, j, k}^{i, j, k}+\omega_{i, j, k}^{i-1, j, k}+\omega_{i+1, j-1, k}^{i, j-1, k}+\omega_{i, j-1, k}^{i-1, j-1, k}\right)} \\
\left.\frac{\partial v}{\partial z}\right|_{i, j-1 / 2, k}= & \frac{\omega_{i, j, k+1}^{i, j, k}\left(v_{i, j, k+1}-v_{i, j, k}\right)+\omega_{i, j, k}^{i, j, k-1}\left(v_{i, j, k}-v_{i, j, k-1}\right)}{\Delta z\left(\omega_{i, j, k+1}^{i, j, k}+\omega_{i, j, k}^{i, j, k-1}+\omega_{i, j-1, k+1}^{i, j-1, k}+\omega_{i, j-1, k}^{i, j-1, k-1}\right)}
\end{aligned}
$$

$$
+\frac{\omega_{i, j-1, k+1}^{i, j-1, k}\left(v_{i, j-1, k+1}-v_{i, j-1, k}\right)+\omega_{i, j-1, k}^{i, j-1, k-1}\left(v_{i, j-1, k}-v_{i, j-1, k-1}\right)}{\Delta z\left(\omega_{i, j, k+1}^{i, j, k}+\omega_{i, j, k}^{i, j, k-1}+\omega_{i, j-1, k+1}^{i, j-1, k}+\omega_{i, j-1, k}^{i, j-1, k-1}\right)},
$$

where,

$$
\begin{aligned}
\omega_{I, J, K}^{L, M} & =\left\{\begin{array}{ll}
1, & s \phi_{I, J, K} \geq 0 \text { and } s \phi_{L, M, N} \geq 0, \\
0, & \text { otherwise }
\end{array} \quad\right. \text { and } \\
s & = \begin{cases}1, & \phi_{i, j-1, k} \geq 0 \text { or } \phi_{i, j, k} \geq 0, \\
-1, & \text { otherwise },\end{cases}
\end{aligned}
$$

$$
\begin{aligned}
\left.\frac{\partial w}{\partial x}\right|_{i, j, k-1 / 2}= & \frac{\omega_{i+1, j, k}^{i, j, k}\left(w_{i+1, j, k}-w_{i, j, k}\right)+\omega_{i, j, k}^{i-1, j, k}\left(w_{i, j, k}-w_{i-1, j, k}\right)}{\Delta x\left(\omega_{i+1, j, k}^{i, j, k}+\omega_{i, j, k}^{i-1, j, k}+\omega_{i+1, j, k-1}^{i, j, k-1}+\omega_{i, j, k-1}^{i-1, j, k-1}\right)} \\
& +\frac{\omega_{i+1, j, k-1}^{i, j, k-1}\left(w_{i+1, j, k-1}-w_{i, j, k-1}\right)+\omega_{i, j, k-1}^{i-1, j, k-1}\left(w_{i, j, k-1}-w_{i-1, j, k-1}\right)}{\Delta x\left(\omega_{i+1, j, k}^{i, j, k}+\omega_{i, j, k}^{i-1, j, k}+\omega_{i+1, j, k-1}^{i, j, k-1}+\omega_{i, j, k-1}^{i-1, j, k-1}\right)}, \\
\left.\frac{\partial w}{\partial y}\right|_{i, j, k-1 / 2}= & \frac{\omega_{i, j+1, k}^{i, j, k}\left(w_{i, j+1, k}-w_{i, j, k}\right)+\omega_{i, j, k}^{i, j-1, k}\left(w_{i, j, k}-w_{i, j-1, k}\right)}{\Delta y\left(\omega_{i, j+1, k}^{i, j, k}+\omega_{i, j, k}^{i, j-1, k}+\omega_{i, j+1, k-1}^{i, j, k-1}+\omega_{i, j, k-1}^{i, j-1, k-1}\right)} \\
& +\frac{\omega_{i, j+1, k-1}^{i, j, k-1}\left(w_{i, j+1, k-1}-w_{i, j, k-1}\right)+\omega_{i, j, k-1}^{i, j-1, k-1}\left(w_{i, j, k-1}-w_{i, j-1, k-1}\right)}{\Delta y\left(\omega_{i, j+1, k}^{i, j, k}+\omega_{i, j, k}^{i, j-1, k}+\omega_{i, j+1, k-1}^{i, j, k-1}+\omega_{i, j, k-1}^{i, j-1, k-1}\right)},
\end{aligned}
$$


where,

$$
\begin{aligned}
\omega_{I, J, K}^{L, M, N} & =\left\{\begin{array}{ll}
1, & s \phi_{I, J, K} \geq 0 \text { and } s \phi_{L, M, N} \geq 0, \\
0, & \text { otherwise }
\end{array} \quad\right. \text { and } \\
s & = \begin{cases}1, & \phi_{i, j, k-1} \geq 0 \text { or } \phi_{i, j, k} \geq 0, \\
-1, & \text { otherwise. }\end{cases}
\end{aligned}
$$

We may now write the viscous coupling terms as

$$
\begin{aligned}
& \left.\frac{\partial\left(\mu \frac{\partial u}{\partial y}\right)}{\partial x}\right|_{i, j, k} \approx \frac{\left.\mu_{i+1 / 2, j, k} \frac{\partial u}{\partial y}\right|_{i+1 / 2, j, k}-\left.\mu_{i-1 / 2, j, k} \frac{\partial u}{\partial y}\right|_{i-1 / 2, j, k}}{\Delta x}, \\
& \left.\frac{\partial\left(\mu \frac{\partial u}{\partial z}\right)}{\partial x}\right|_{i, j, k} \approx \frac{\left.\mu_{i+1 / 2, j, k} \frac{\partial u}{\partial z}\right|_{i+1 / 2, j, k}-\left.\mu_{i-1 / 2, j, k} \frac{\partial u}{\partial z}\right|_{i-1 / 2, j, k}}{\Delta x}, \\
& \left.\frac{\partial\left(\mu \frac{\partial v}{\partial x}\right)}{\partial y}\right|_{i, j, k} \approx \frac{\left.\mu_{i, j+1 / 2, k} \frac{\partial v}{\partial x}\right|_{i, j+1 / 2, k}-\left.\mu_{i, j-1 / 2, k} \frac{\partial v}{\partial x}\right|_{i, j-1 / 2, k}}{\Delta y}, \\
& \left.\frac{\left.\partial\left(\mu \frac{\partial v}{\partial z}\right)\right|_{i, j, k} \approx \frac{\left.\mu_{i, j+1 / 2, k} \frac{\partial v}{\partial z}\right|_{i, j+1 / 2, k}-\left.\mu_{i, j-1 / 2, k} \frac{\partial v}{\partial z}\right|_{i, j-1 / 2, k}}{\Delta y}}{\Delta z}\right|_{i, j, k} \approx \frac{\left.\mu_{i, j, k+1 / 2} \frac{\partial w}{\partial x}\right|_{i, j, k+1 / 2}-\left.\mu_{i, j, k-1 / 2} \frac{\partial w}{\partial x}\right|_{i, j, k-1 / 2}}{\Delta z} \\
& \left.\frac{\partial\left(\mu \frac{\partial w}{\partial y}\right)}{\partial z}\right|_{i, j, k} \approx \frac{\left.\mu_{i, j, k+1 / 2} \frac{\partial w}{\partial y}\right|_{i, j, k+1 / 2}-\left.\mu_{i, j, k-1 / 2} \frac{\partial w}{\partial y}\right|_{i, j, k-1 / 2}}{\Delta z} .
\end{aligned}
$$

\subsection{Justification for New Method for Discretizing the Viscous Coupling Terms}

In Sussman and Ohta [3] the discretization of the coupling terms was done in a manner so that the gas velocity would not be included in the discretization of the viscous force terms in the case $\mu_{G}=0$. This restriction sacrificed consistency in the discretization of the coupling terms near the interface. We believe that this inconsistency is the reason why we have encountered instability for some problems when treating the coupling terms explicitly. In order to illustrate the problem, and in order to illuminate the reasoning behind our solution, we refer the reader to Fig. 1. If we were to implement the method described by Sussman and Ohta [3], one would define the following nodal viscosities:

$$
\boldsymbol{\mu}_{i+1 / 2, j+1 / 2}=0, \quad \boldsymbol{\mu}_{i+1 / 2, j-1 / 2}=\boldsymbol{\mu}^{L} .
$$

The resulting discretization for the following coupling term $\frac{\partial\left(\mu u_{y}\right)}{\partial x}$ would be,

$$
\begin{aligned}
\left(\frac{\partial\left(\mu u_{y}\right)}{\partial x}\right) \approx & \left(\mu_{i+1 / 2, j-1 / 2}\left(u_{y}\right)_{i+1 / 2, j-1 / 2}+(0)\left(u_{y}\right)_{i+1 / 2, j+1 / 2}\right. \\
& \left.-\mu_{i-1 / 2, j-1 / 2}\left(u_{y}\right)_{i-1 / 2, j-1 / 2}-\mu_{i-1 / 2, j+1 / 2}\left(u_{y}\right)_{i-1 / 2, j+1 / 2}\right) / 2 \Delta x .
\end{aligned}
$$

The first two terms of (3.4) combines to be an inconsistent discretization of the quantity,

$$
\mu^{L}\left(\frac{\partial u}{\partial y}\right)_{i+1 / 2, j}
$$


Fig. 1 Stencil for calculating the viscous force term at cell $(i, j)$. For the zero gas viscosity case, the quantity in the upper right hand corner should not be included but at the same time, the discretization of the components of the stress tensor should be consistent in the limit that the mesh size goes to zero

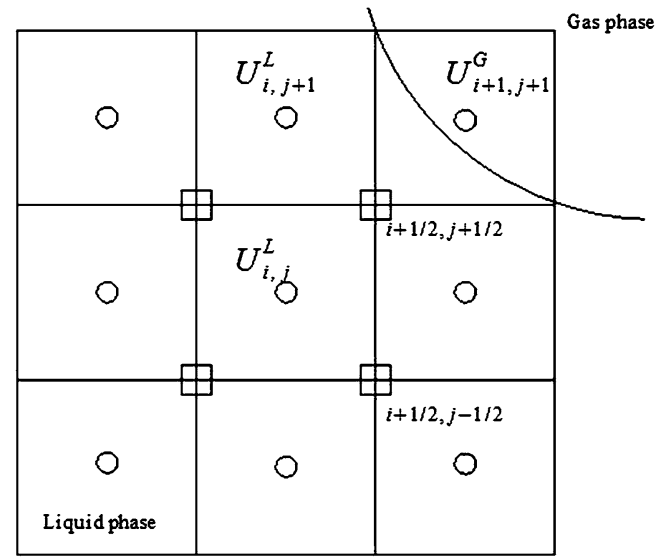

The level set function does not change sign between cells $(i, j)$ and $(i+1, j)$ so one should consistently define all components of the rate of deformation tensor that are located at the face $(i+1 / 2, j)$. In particular the previous method is off by a factor of two. Instead we propose the following discretization for the coupling term, $\frac{\partial\left(\mu u_{y}\right)}{\partial x}$,

$$
\begin{aligned}
& \left.\frac{\partial\left(\mu \frac{\partial u}{\partial y}\right)}{\partial x}\right|_{i, j}=\frac{\mu_{i+1 / 2, j}\left(\frac{\partial u}{\partial y}\right)_{i+1 / 2, j}-\mu_{i-1 / 2, j}\left(\frac{\partial u}{\partial y}\right)_{i-1 / 2, j}}{\Delta x} \\
& =\frac{\mu^{L} \frac{1}{3}\left(\frac{u_{i, j}-u_{i, j-1}}{\Delta y}+\frac{u_{i+1, j}-u_{i+1, j-1}}{\Delta y}+\frac{u_{i, j+1}-u_{i, j}}{\Delta y}\right)-\mu^{L}\left(\frac{u_{i, j+1}-u_{i, j-1}+u_{i-1, j+1}-u_{i-1, j-1}}{4 \Delta y}\right)}{\Delta x} .
\end{aligned}
$$

\section{Positive Preserving Update of the Configuration Tensor and the Calculation of the Viscoelastic Force Term}

The equation for the configuration tensor,

$$
\frac{\partial \mathbf{A}}{\partial t}+\mathbf{u} \cdot \nabla \mathbf{A}=\mathbf{A} \cdot(\nabla \mathbf{u})^{T}+\nabla \mathbf{u} \cdot \mathbf{A}-\frac{f(R)}{\lambda}(\mathbf{A}-\boldsymbol{I})
$$

is solved in the following steps:

1 .

$$
A_{i, j}^{*}=A^{n}(\vec{x}-\vec{u} \Delta t) .
$$

Equation (4.1) corresponds to a semi-Lagrangian approach in which we use linear interpolation in order to evaluate $A^{n}(\vec{x}-\vec{u} \Delta t)$. Linear interpolation guarantees that $A^{*}$ is positive definite if $A^{n}$ is.

2. $A_{i, j}^{* *}=S A_{i, j}^{*} S^{T}, S=I+\Delta t\left[\nabla U^{L}\right]_{i, j}^{n}$.

3.

$$
A_{i, j}^{n+1}=\frac{\Delta t \frac{f(R)}{\lambda}}{1+\Delta t \frac{f(R)}{\lambda}} I+\frac{1}{1+\Delta t \frac{f(R)}{\lambda}} A_{i, j}^{* *} .
$$

4. Extrapolate $A_{i, j}^{n+1}$ from the liquid regions $\left(\phi_{i, j} \geq 0\right)$ into the gas regions $\left(\phi_{i, j}<0\right)$ using "constant" extrapolation. The extrapolation is carried out by overwriting $A_{i, j}^{n+1}$ in a 
gas cell with the corresponding value from the nearest liquid cell. Constant extrapolation guarantees that the extrapolated configuration tensor remains positive definite if the original was positive definite as well.

The viscoelastic force term, $\nabla \cdot\left(\frac{\tilde{\eta}_{P}}{\lambda} f(R) A\right)$, is calculated in the following steps:

(a) extrapolate the tensor, $B_{i, j}=\frac{\tilde{\eta}_{P}}{\lambda} f(R)_{i, j} A_{i, j}$, from cell centers to cell faces. E.g.

$$
\begin{aligned}
& B_{i+1 / 2, j}=\frac{\left(\tilde{\eta}_{p}\right)_{i+1 / 2, j}}{\lambda} \frac{(f(R) A)_{i+1, j}+(f(R) A)_{i, j}}{2}, \quad \text { where } \\
& \left(\tilde{\eta}_{P}\right)_{i+1 / 2, j}= \begin{cases}\eta_{P}, & \phi_{i+1, j} \geq 0 \text { and } \phi_{i, j} \geq 0, \\
0, & \text { otherwise. }\end{cases}
\end{aligned}
$$

(b) The components of the viscoelastic force vector are then written as:

$$
\left(L_{i, j}^{\mathrm{viscoelastic}}\right)_{\gamma}=\frac{\left(B_{i+1 / 2, j}\right)_{\gamma, 1}-\left(B_{i-1 / 2, j}\right)_{\gamma, 1}}{\Delta x}+\frac{\left(B_{i, j+1 / 2}\right)_{\gamma, 2}-\left(B_{i, j-1 / 2}\right)_{\gamma, 2}}{\Delta y} .
$$

\subsection{Proof of the Positive Definite Preserving Property for the Configuration Tensor} Update Step

Each of the steps one thru four in Sect. 4 above preserves the configuration tensor as a positive definite symmetric matrix. This fact can be proved so long as the following stability constraint is satisfied,

$$
\Delta t<\frac{1}{2 d} \frac{\Delta x}{\max |U|},
$$

where $d$ is the dimension of the problem ( 2 or 3 ). We first consider step 1 above. Without loss of generality, we consider step 1 (4.1) in one dimension with positive velocity $u$. Then one has

$$
A_{i}^{*}=A^{n}(x-u \Delta t)=\frac{u \Delta t}{\Delta x} A_{i-1}^{n}+\left(1-\frac{u \Delta t}{\Delta x}\right) A_{i}^{n} .
$$

The stability constraint (4.2) insures that the coefficients of $A$ in (4.3) are positive which in turn insures that $A^{*}$ is positive definite symmetric since the linear combination of positive definite symmetric matrices, with positive coefficients, is also positive definite symmetric. In step 2 of Sect. 4 above, the stability constraint (4.2) insures that the matrix,

$$
S=I+\Delta t\left[\nabla U^{L}\right]_{i, j}^{n},
$$

is nonsingular. Since $S$ is nonsingular, and $A^{*}$ is positive definite symmetric, this implies that the matrix $A^{* *}=S A^{*} S^{T}$ is also a positive definite symmetric matrix. In step 3 of Sect. 4 above, we have the linear combination of two positive definite symmetric matrices, with positive coefficients, which insures that $A^{n+1}$ is also positive definite symmetric. Finally, the extrapolation step (step 4) preserves the extrapolated values of $A^{n+1}$ as positive definite symmetric since we are implementing a piecewise constant extrapolation algorithm. We note that for our choice of $f(R)$ in (1.2), we have to treat the special case when $\operatorname{trace}(A) \geq L^{2}$; in this case we assume a relaxation time of zero and therefore replace the configuration tensor $A$ with the identity matrix $I$. 


\section{Matrix Solver}

The equations resulting from the calculations of the viscous force terms or the pressure projection steps can be written generally as,

$$
\alpha(x) p+\beta \nabla \cdot(A(x, y) \nabla p)=f(x, y)+\alpha(x) q+\beta \nabla \cdot V,
$$

where $A$ is a diagonal matrix. We discretize (5.1) as,

$$
\alpha_{i j} p_{i j} \beta(D G p)_{i j}=f_{i j}+\alpha_{i j} q_{i j}+\beta(D V)_{i j}
$$

where

$$
\begin{aligned}
& (G p)_{i+1 / 2, j}=\left(A^{11}\right)_{i+1 / 2, j} \frac{p_{i+1, j}-p_{i j}}{\Delta x}, \quad(G p)_{i, j+1 / 2}=\left(A^{22}\right)_{i, j+1 / 2} \frac{p_{i, j+1}-p_{i j}}{\Delta y}, \\
& (D V)_{i, j}=\frac{u_{i+1 / 2, j}-u_{i-1 / 2, j}}{\Delta x}+\frac{v_{i, j+1 / 2}-v_{i, j-1 / 2}}{\Delta y} .
\end{aligned}
$$

We solve (5.2) with a combination of the multigrid method and the mutligrid preconditioned conjugate gradient method (MGPCG) [21]. The adaptive composite solver will converge to any specified tolerance $\varepsilon$, as long as we compute the solution on each level (using MGPCG) to a tolerance of $\varepsilon \times 10^{-2}$ each time the outer multigrid solver visits a level. Our Multigrid+MGPCG algorithm for solving (5.2) follows the same "V-cycle" procedure as outlined by Briggs, et al. [22]:

Put (5.2) in residual correction form:

For $l=0 \ldots l_{\max }$

$$
\begin{aligned}
& V^{l}=V^{l}-G p_{\text {predict }}^{l} \\
& q^{l}=q^{l}-p_{\text {predict }}^{l} \\
& p^{l}=p_{\text {predict }}^{l}
\end{aligned}
$$

\section{EndFor}

Then, call recursive subroutine $\operatorname{MV}(l)$ :

\section{subroutine $\mathrm{MV}(\boldsymbol{l})$}

1. if $l<l_{\max }$ then restrict $V^{l+1}$ to level $l$

2. $R^{l}=f^{l}+\alpha q^{l}+\beta D V^{l}$

3. if $l<l_{\max }$ then restrict $R^{l+1}$ to level $l$

4. $V_{\text {save }}^{l}=V^{l}, q_{\text {save }}^{l}=q^{l}, R_{\text {save }}^{l}=R^{l}$

5. $p_{\text {cor }}^{l}=0$

6. Iterate until the residual on level $l$ is less than the tolerance $\varepsilon \times 10^{-2}$.

Iterate using MGPCG,

$$
\alpha p_{\text {cor }}^{l}+\beta D G p_{\text {cor }}^{l}=R_{\text {save }}^{l}
$$

7. $R^{l}=R_{\text {save }}^{l}-\alpha p_{\text {cor }}^{l}-\beta D G p_{\text {cor }}^{l}, V^{l}=V^{l}-G p_{\text {cor }}^{1}, q^{l}=q^{l}-p_{\text {cor }}^{l}$

8. if $l>0$ then $\left\{\begin{array}{l}\text { call } M V(l-1) \\ \text { prolongate } p_{\text {cor }}^{l-1} \text { to level } l, p_{\text {cor }}^{l}=p_{\text {cor }}^{l}+I_{l-1}^{l} p_{\text {cor }}^{l-1}\end{array}\right.$

9. Iterate until the residual on level $l$ is less than the tolerance $\varepsilon \times 10^{-2}$.

Iterate using MGPCG,

$$
\alpha p_{\mathrm{cor}}^{l}+\beta D G p_{\mathrm{cor}}^{l}=R_{\mathrm{save}}^{l}
$$

10. $V^{l}=V_{\mathrm{save}}^{l}-G p_{\mathrm{cor}}^{l}, q^{l}=q_{\mathrm{save}}^{l}-p_{\mathrm{cor}}^{l}, p^{l}=p^{l}+p_{\mathrm{cor}}^{l}$ 


\section{Overall Numerical Algorithm for Two-Phase Viscoelastic/Viscous Flow}

Prior to each time step, we are given a liquid velocity, $U^{L, n}$, and a total velocity $U^{n} . U^{L, n}$ corresponds to $U^{n}$ except on gas faces, where we replace the gas velocity in $U^{L, n}$ with the extrapolated liquid velocity. $U^{L, n}$ is then used to calculate the nonlinear advective terms in the liquid and also used to advance the free surface.

Prior to each time step, we are also given a level set function, $\varphi^{n}$ and a volume-offluid function $F^{n}$. The level set function and the volume-of-fluid function are stored at cell centers. The velocity is stored at both cell centers and face-centers.

An outline of our numerical algorithm is as follows:

1. CLSVOF; interface advection [1]:

$$
\varphi_{i j}^{n+1}=\varphi_{i j}^{n}-\Delta t\left[U^{L} \cdot \nabla \phi\right]_{i j}, \quad F_{i j}^{n+1}=F_{i j}^{n}-\Delta t\left[U^{L} \cdot \nabla F\right]_{i j} .
$$

2. Calculate (cell centered) advective force terms:

$$
A_{i j}^{L}=\left[U^{L} \cdot \nabla U^{L}\right]_{i j}^{n}, \quad A_{i j}=[U \cdot \nabla U]_{i j}^{n} .
$$

The nonlinear advective terms are discretized using upwind, second order Van-Leer slop limiting $[1,23]$.

3. Update the configuration tensor (viscoelastic flows):

$$
A_{i, j}^{n+1}=\frac{\Delta t \frac{f(R)}{\lambda} I+\left(S A^{n}(\vec{x}-\vec{u} \Delta t) S^{T}\right)_{i, j}}{1+\Delta t \frac{f(R)}{\lambda}}, \quad S=I+\Delta t \nabla U .
$$

Please see Sect. 4 for details that describe the discretization of (6.1).

4. Calculate (cell centered) semi-implicit viscous forces and explicit viscoelastic forces:

$$
\begin{aligned}
U_{i, j}^{n}= & \left\{\begin{array}{ll}
U_{i, j}^{L, n}, & \phi_{i, j} \geq 0, \\
U_{i, j}^{n}, & \phi_{i, j}<0,
\end{array} \quad A_{i j}= \begin{cases}A_{i, j}^{L}, & \phi_{i, j} \geq 0, \\
A_{i, j}, & \phi_{i, j}<0,\end{cases} \right. \\
& U_{i, j}^{*}=U_{i, j}^{n}+\Delta t\left(-A_{i, j}+L_{i, j}^{\text {viscoelastic }}+g \hat{z}\right), \\
& \rho \frac{U^{* *}-U^{*}}{\Delta t}=L^{* *, \text { uncoupled }}+L^{* \text {, uncoupled }} .
\end{aligned}
$$

The term $L^{* * \text { uncoupled }}$ represents the uncoupled viscous force terms which are handled implicitly, and the term $L^{* \text {,coupled }}$ represents the coupled viscous force terms which are handled explicitly.

5. Interpolate cell centered forces to face centered forces and calculate the face centered surface tension force:

$$
\begin{aligned}
V_{i+1 / 2, j}= & U_{i+1 / 2, j}^{n}+\Delta t\left(-A_{i+1 / 2, j}+\frac{2}{\rho_{i+1, j}+\rho_{i, j}}\left(L_{i+1 / 2, j}+L_{i+1 / 2, j}^{\text {viscoelastic }}\right)\right. \\
& \left.-\left[\frac{\sigma \kappa \nabla H}{\rho}\right]_{i+1 / 2, j}+g \hat{z}\right) .
\end{aligned}
$$

The Surface tension term, $\frac{\sigma \kappa_{i+1 / 2, j}(\nabla H)_{i+1 / 2, j}}{\rho_{i+1 / 2, j}}$, is discretized as

$$
\begin{gathered}
\sigma \kappa_{i+1 / 2, j} \frac{H\left(\phi_{i+1, j}\right)-H\left(\phi_{i, j}\right)}{\rho_{i+1 / 2, j} \Delta x}, \quad \text { where } H(\phi)=\left\{\begin{array}{ll}
1, & \phi \geq 0, \\
0, & \phi<0,
\end{array} \quad\right. \text { and } \\
\rho_{i+1 / 2, j}=\rho_{L} \theta_{i+1 / 2, j}+\rho_{G}\left(1-\theta_{i+1 / 2, j}\right) .
\end{gathered}
$$


Table 1 Comparison of computational results with analytical solution for $R e \ll 1$

\begin{tabular}{lll}
\hline & Previous & New \\
\hline Computational Re number & 0.0524 & 0.0524 \\
Analytical solution by Hadamard-Rybczynski & 0.0534 & 0.0534 \\
\hline
\end{tabular}

The discretization of the height fraction $\theta_{i+1 / 2, j}$ is described by (3.2). The curvature $\kappa_{i+1 / 2, j}$ is computed with second-order accuracy directly from the volume fractions as described in [24] or [1].

6. Implicit pressure projection step:

$$
\nabla \cdot \frac{\nabla p}{\rho}=\nabla \cdot V, \quad U_{i+1 / 2, j}^{n+1}=V_{i+1 / 2, j}-\left[\frac{\nabla p}{\rho}\right]_{i+1 / 2, j} .
$$

We solve the resulting linear system using a composite solver for an adaptive hierarchy of grids [25] (see also Sect. 5).

7. Liquid velocity extrapolation; assign $U_{i+1 / 2, j}^{L, n+1}=U_{i+1 / 2, j}^{n+1}$ and then extrapolate $U_{i+1 / 2, j}^{L, n+1}$ into the gas region.

8. Interpolate face centered velocity to cell centered velocity:

$$
U_{i, j}^{L, n+1}=\frac{1}{2}\left(U_{i+1 / 2, j}^{L, n+1}+U_{i-1 / 2, j}^{L, n+1}\right), \quad U_{i, j}^{n+1}=\frac{1}{2}\left(U_{i+1 / 2, j}^{n+1}+U_{i-1 / 2, j}^{n+1}\right) .
$$

\section{Results}

7.1 Spherical Bubble or Drop with Dominant Viscous Effects and Large Viscosity Jump: Comparison with Analytical and Experimental Results

For a spherical bubble, drop and particle freely moving relative to a fluid of infinite extent with a steady velocity with $R e \ll 1.0$, the analytical solution derived by HadamardRybczynsk [26, 27] is well-known as follows:

$$
R e=\frac{E o^{1.5}}{6 M^{0.5}}\left(\frac{1+\kappa}{2+3 \kappa}\right)
$$

Here, $E o$ is the Eotvos number, $M$ denotes the Morton number and $\kappa\left(=\mu_{\mathrm{D}} / \mu_{\mathrm{C}}\right)$ is the viscosity ratio. Equation (7.1) reduces to $R e=\frac{E o^{1.5}}{12 M^{0.5}}$ as $\kappa \rightarrow 0$ and becomes $\frac{E o^{1.5}}{18 M^{0.5}}$ for $\kappa \rightarrow \infty$. We show results for a spherical bubble with low Reynolds number. The computational condition is $\log M=2.85, E o=8.67$ which is an experimental condition performed by Bhaga and Weber [28]. Although they examined bubble rise dynamics, in this computation, we changed to a density ratio $\lambda\left(=\rho_{\mathrm{D}} / \rho_{\mathrm{C}}\right)=0.95$ and $\kappa=100$ to clearly specify a problem with a large viscosity jump. The numerical simulations were performed on a 3-d axisymmetric computational domain with a domain length of $R=4 D$ ( $r$-direction) and a domain height of $Z=6 D$ ( $z$-direction). In the computation, one mesh size on the finest level grid was set to $\Delta x=0.0104$ in terms of the dimensionless bubble/drop diameter $(D=1.0)$. Comparison of computational results with analytical solution is indicated in Table 1 . The error between our computational results and analytical solution is only about $2 \%$. We reiterate that the fluid properties for this problem exhibit very large viscosity. 
Fig. 2 Left: previous method, right: present method, $\log M=2.93, E o=116$

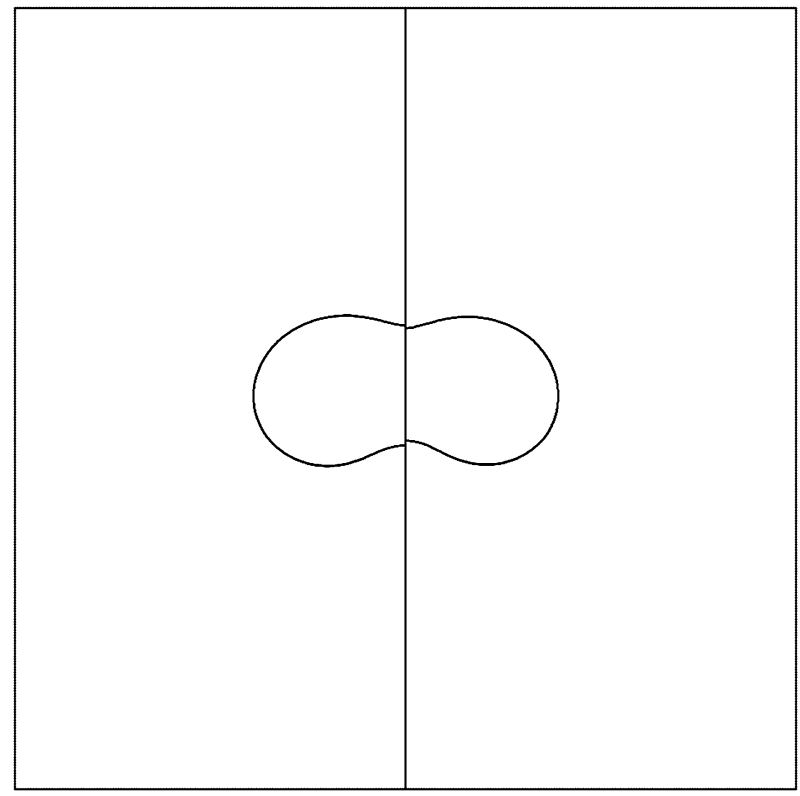

We then recomputed the previous example $(\lambda=0.95, \kappa=100)$, except with $\log M=$ 2.93 and $E o=116$; this test was also performed by Bhaga and Weber [28]. Figure 2 shows a comparison of results using our previous method from [3] and the new methodology described in this paper. Computational conditions are the same as the case for low Re number. In Fig. 2, the left side is the previous method and the right side corresponds to the new method.

\subsection{Drop Formation in a Miscible Viscous Liquid}

Figure 3 corresponds to the drop formation process in a miscible viscous liquid. Bright [29] injected n-decane into water from a submerged nozzle with $d=1.6 \times 10^{-3} \mathrm{~m}$, and he investigated the drop formation process in the system $(\lambda=0.7327$ and $\kappa=0.90)$. Richards et al. [30] numerically calculated the Bright's experimental result on the drop formation process using the SOLA-VOF method [31] with the continuum surface force (CFS) model [32]. In their computation, Richards et al. [30] used the value of the interfacial tension experimentally estimated by Johnson and Dettre [33] instead of the interfacial tension measured by Bright [29]. Our computational conditions for the physical properties follow the paper of Richards et al. [30]. The computations were carried out in a 3-d axisymmetric computational domain with a domain length of $R=16 d$ ( $r$-direction) and a domain height of $Z=32 d$ ( $z$ direction). Also, one mesh size on the finest level grid was $\Delta x=0.0125$ in terms of the dimensionless diameter of nozzle (=1.0). In Fig. 3, the left side is the previous method [3] and the right side corresponds to the new method. We have confirmed that the appearance of the drop formation reproduced numerically agrees with the experimental result.

\subsection{Bubble Formation; Injection of High Speed Gas into Liquid}

In test problems with fluids that have a large viscosity, such as the test problem described in Sect. 7.1 above, a fully implicit treatment of the coupled viscous terms would lead to 
Fig. 3 Drop formation in a viscous liquid. Left: old method, right: new method

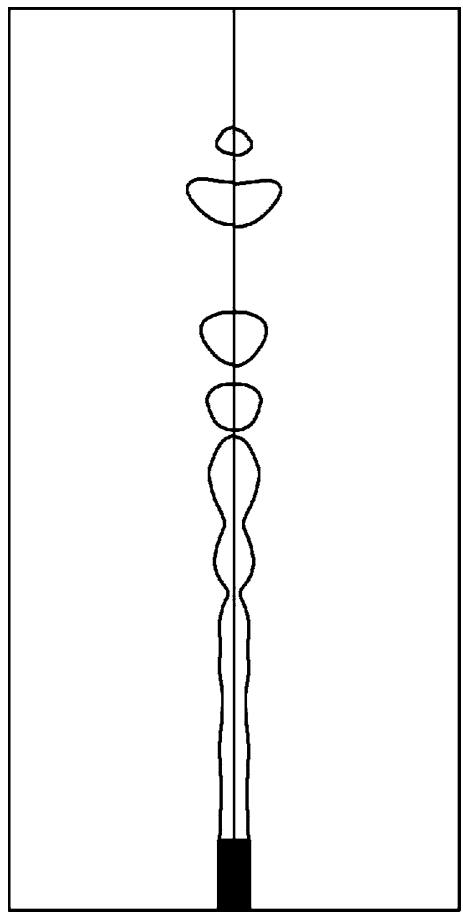

Table 2 Grid resolution study for bubble formation problem. Relative errors are displayed. Inflow velocity is $0.9 \mathrm{~m} / \mathrm{s}$; density ratio $=1015$, viscosity ratio $=70000$

\begin{tabular}{|c|c|c|c|}
\hline Eff. Grid Resolution & $64 \times 128$ & $128 \times 256$ & $256 \times 512$ \\
\hline Interface Error & & 0.74 & 0.45 \\
\hline Gas Velocity Error & & 1.44 & 0.65 \\
\hline
\end{tabular}

a matrix system that could not be solved via a standard matrix iterative approach. On the other hand we have found that, for the bubble formation problem, the explicit treatment of the viscous coupling terms, as described by [3], leads to instabilities. Here, we perform a grid refinement study using our new approach for a problem that we had previously failed to compute using the approach described in [3]. The physical properties of the system, together with the inflow velocity condition, are given by the following dimensionless quantities,

$$
\begin{aligned}
\operatorname{Re}_{G} & =\frac{\rho_{G} V d}{\mu_{G}}=99.2, \quad W e_{G}=\frac{\rho_{G} V^{2} d}{\sigma}=0.024, \quad F r=\frac{V^{2}}{g d}=46.6, \\
\frac{\rho_{L}}{\rho_{G}} & =1015, \quad \frac{\mu_{L}}{\mu_{G}}=70000 .
\end{aligned}
$$

The relative errors in Table 1 indicate that we have first order convergence for this problem using our new treatment for the viscous coupling terms. 
Fig. 4 Bubble formation, left: $64 \times 128$, middle: 1 level of adaptivity, right: 2 levels of adaptivity; inflow velocity is $0.9 \mathrm{~m} / \mathrm{s}$; density ratio $=1015$, viscosity ratio $=70000$
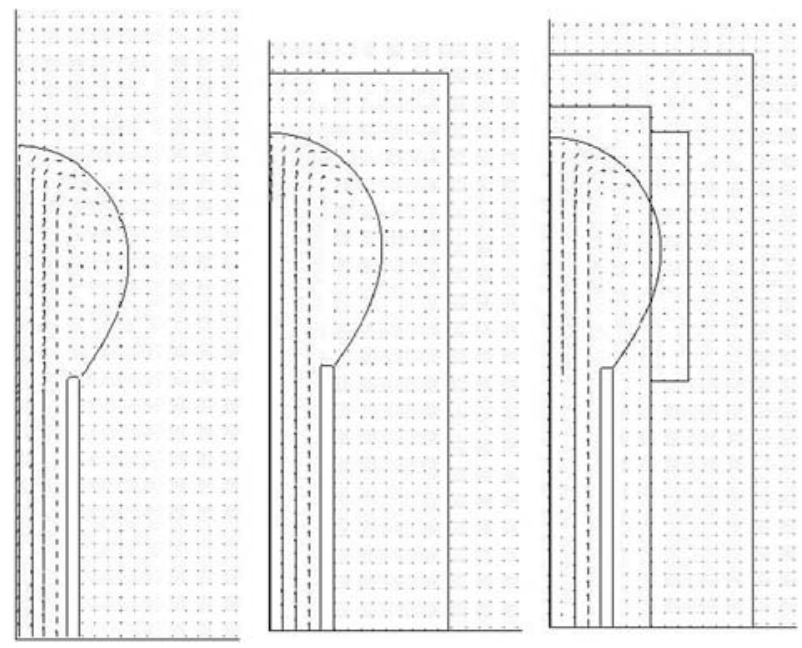
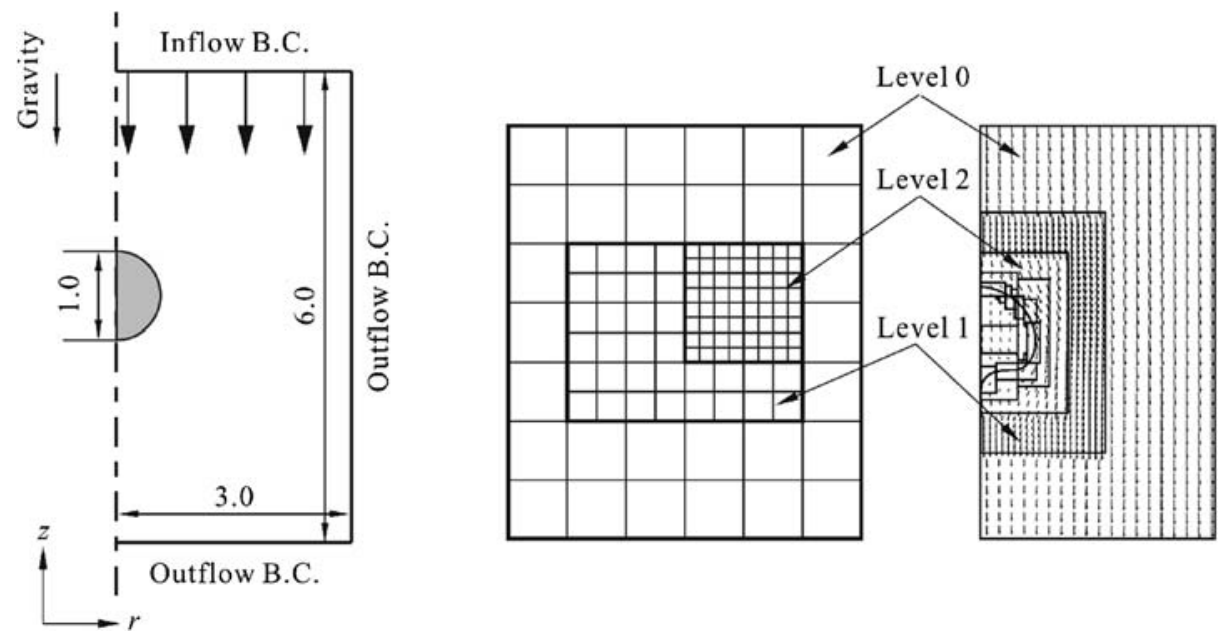

Fig. 5 Computational setup; dynamic adaptive gridding used around the viscoelastic bubble

\subsubsection{Viscoelastic Bubbles}

We consider a gas-liquid system with the physical properties defined by the Morton number $M\left(=g \eta_{S}^{4}\left(\rho_{S}-\rho_{G}\right) / \rho_{S}^{2} \sigma^{3}\right)=78$, the density ratio $\rho_{S} / \rho_{G}=845$ and the viscosity ratio $\eta_{S} / \eta_{G}=55000$. We shall investigate the effect of FENE-CR model parameters given these physical properties. The computational domain for the $2 \mathrm{~d}$-axisymmetric computations is shown schematically in Fig. 5. In terms of the dimensionless diameter of bubble $d=1.0$, the computational domain has an $r$-directional dimensionless length $R=3.0$ and a $z$-directional dimensionless height $H=7.5$. In our computations, as initial conditions, a spherical bubble was artificially imposed at $H=4.5$. The physical boundary conditions were as follows: inflow at the top wall, outflow boundaries at the bottom and right walls, and reflecting boundary conditions at the axisymmetric center $(r=0)$. 
Fig. 6 Bubble shapes depending on the Deborah number
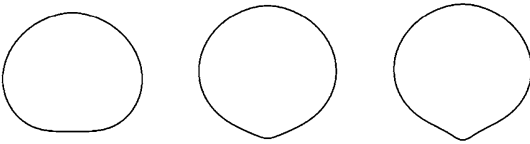

Newtonian

$D e=5.0$
$E o=19.6, L=5.0, c=0.1$
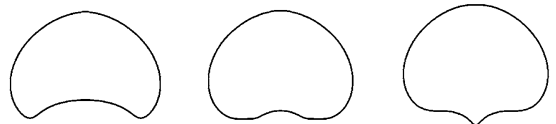

$D e=5.0$

$$
\begin{aligned}
\text { Newtonian } \quad D e & =1.0 \quad D e= \\
E o=78.3, L & =10.0, c=0.1
\end{aligned}
$$

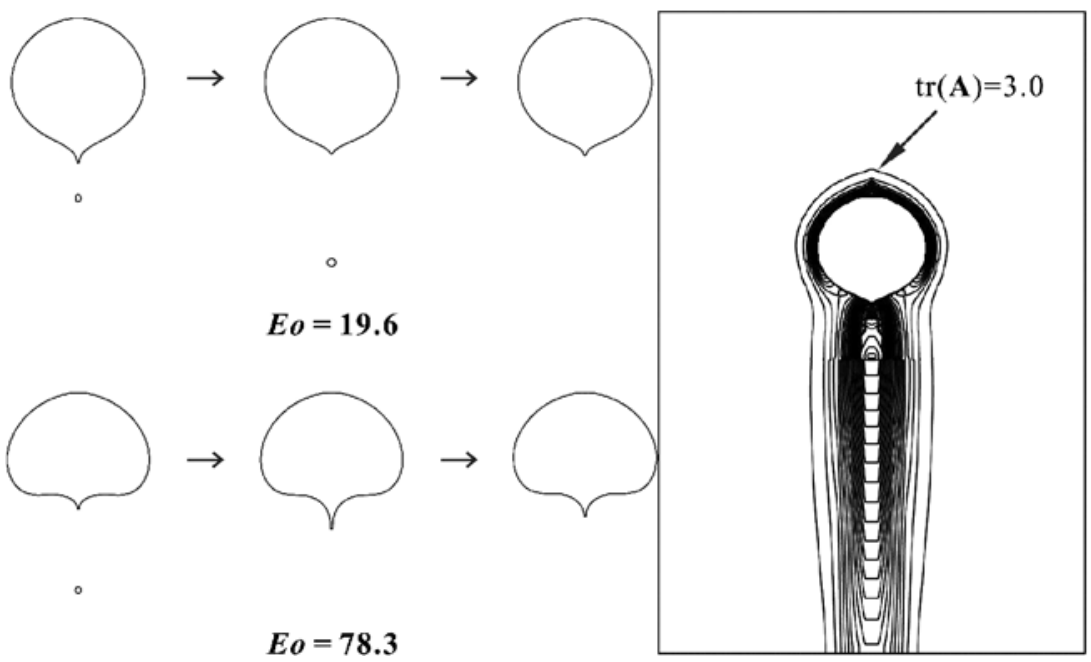

Fig. 7 Left: transient dynamics of bubble for $L=20, c=0.1, D e=5.0$. Right: distribution of $\operatorname{tr}(A)$ for $E o=19.6, L=10, c=0.1, D e=5.0$

Figure 5 is a schematic drawing of the AMR system. The left side of Fig. 5 shows an example of the grid structure and the right side of Fig. 5 shows a computational example of a $2 \mathrm{~d}$-axisymmetric bubble rising. The mesh hierarchy is composed of different levels of refinement ranging from coarsest $\left(\ell=0\right.$, we label as "Level 0 ".) to finest $\left(\ell=\ell_{\max }\right)$, and the refinement ratio between levels is 2; i.e. $\Delta x^{\ell+1}=0.5 \Delta x^{\ell}$. In Fig. 5, five levels (Level $0-4)$ are indicated. In the computations, one mesh size on the finest adaptive level $\Delta x$ fine was about 0.0078 in terms of $d=1.0$. The value $\Delta x^{\text {fine }}=0.0078$ was adopted as a result of a mesh refinement study for the stiffest flow condition. This means there are (effectively) about 85 cells per bubble diameter.

Figure 6 shows bubble shapes depending on the Deborah number $D e(=V \lambda / D, V$ : characteristic velocity (bubble rise speed), $D$ : bubble diameter). The upper figures are obtained under the condition of the Eötvös number $E o\left(=g\left(\rho_{S}-\rho_{G}\right) D^{2} / \sigma\right)=19.6, L=5.0, c=0.1$ and the lower figures use $E o=78.3, L=10.0, c=0.1$. As a basis of comparison, the results for the corresponding Newtonian bubble are indicated together with the viscoelastic results. For the largest $D e$ number, bubbles have the well-known sharp cusp-like trailing 
edge. Especially, regardless of the largely dimpled Newtonian bubble for $E o=78.3$, it is surprising that the bubble for $L=10.0, c=0.1, D e=5.0$ has a very long sharp cusp. This result indicates that our computational method can capture largely deformed shapes having large curvature. It is verified that viscoelastic effects (cusp shape) emerge notably as the De number becomes larger. Noh et al. [34] gave good physical explanations about the effect of the parameters of the FENE-CR model, and our results support their observations. Figure 7 shows transient dynamics of bubble motion for the condition of $L=20, c=0.1, D e=5.0$. In this case, the bubble trailing edge is pulled out due to the strong viscoelastic effect (viscoelastic stress). Consequently, the pulling motion leads to the local breakup of the bubble at the trailing edge. As an example of the $\operatorname{tr}(\mathbf{A})$ profile, the distribution $\operatorname{of} \operatorname{tr}(\mathbf{A})$ for $E o=19.6$, $L=10, c=0.1, D e=5.0$ is shown as well in Fig. 7. $\operatorname{tr}(\mathbf{A})$ is a measure of the degree of polymer extension. In Fig. 7, a contour plot of $\operatorname{tr}(\mathbf{A})$ is shown in which 40 contours are plotted ranging from the maximum trace $(\operatorname{tr}(\mathbf{A})=95.0)$ to the minimum trace $(\operatorname{tr}(\mathbf{A})=3.0)$. The outer contour corresponds to the minimum value. As is clear in Fig. 7, the value of $\operatorname{tr}(\mathbf{A})$ is relatively large near the interface and that the local maximums of $\operatorname{tr}(\mathbf{A})$ are near the top and bottom surfaces. Due to these viscoelastic stresses, it can be recognized that the trailing cusp at the bottom is formed and the vertical bubble length increases.

\section{Conclusions}

We have tested two improvements for discretizing the viscosity force terms, and viscoelastic force terms as they appear in the Navier-Stokes equations for two-phase flows. These improvements preserve the property of our sharp interface approach in that solutions of the two-phase problem approach solutions of the corresponding one-phase problem in the limit of zero gas density and zero gas viscosity. These two improvements enable us to robustly calculate viscous and viscoelastic two-phase flows over a very large range of physical properties of the flow. For example, in Sect. 7.1 we have validated our approach for problems where $R e \ll 1$. In Sect. 7.3, we validated our new approach for a bubble formation problem with a relatively large Reynolds number: $R e_{G}=99.2$. The method described by [3] becomes unstable for the bubble formation problem. As illustrated by Fig. 3 and Fig. 7, our coupled level-set and volume-of-fluid approach for representing and updating the interface can accurately handle sharp corners, and changes in interface topology.

Acknowledgements We extend a warm thank you to S. Yamaguchi (viscosity effect), D. Kikuchi (bubble/drop formation), and K. Onodera (viscoelastic bubble) for their contributions to the results of this paper. Our work is supported in part by NSF grant number 0242524 (U.S. Japan Cooperative Science) and JSPS.

\section{References}

1. Sussman, M., Smith, K.M., Hussaini, M.Y., Ohta, M., Zhi-Wei, R.: A sharp interface method for incompressible two-phase flows. J. Comput. Phys. 221(2), 469-505 (2007)

2. Jimenez, E., Sussman, M., Ohta, M.: A computational study of bubble motion in Newtonian and viscoelastic fluids. Fluid Dyn. Mater. Process. 1(2), 97-108 (2005)

3. Sussman, M., Ohta, M.: Improvements for calculating two-phase bubble and drop motion using an adaptive sharp interface method. Fluid Dyn. Mater. Process. 3(1), 21-36 (2007)

4. Kang, M., Fedkiw, R., Liu, X.-D.: A boundary condition capturing method for multiphase incompressible flow. J. Sci. Comput. 15 (2000)

5. Fedkiw, R.P., Aslam, T., Merriman, B., Osher, S.: A non-oscillatory Eulerian approach to interfaces in multimaterial flows (the Ghost fluid method). J. Comput. Phys. 152(2), 457-492 (1999) 
6. Liu, X.-D., Fedkiw, R.P., Kang, M.: A boundary condition capturing method for Poisson's equation on irregular domains. J. Comput. Phys. 160(1), 151-178 (2000)

7. Li, J., Renardy, Y.Y., Renardy, M.: Numerical simulation of breakup of a viscous drop in simple shear flow through a volume-of-fluid method. Phys. Fluids 12(2), 269-282 (2000)

8. Hong, J.-M., Shinar, T., Kang, M., Fedkiw, R.: On boundary condition capturing for multiphase interfaces. J. Sci. Comput. 31, 99-125 (2007)

9. Rasmussen, N., Enright, D., Nguyen, D., Marino, S., Sumner, N., Geiger, W., Hoon, S., Fedkiw, R.: Directable photorealistic liquids. In: Eurographics/ACM SIGGRAPH Symposium on Computer Animation, 2004

10. Yu, J.-D., Sakai, S., Sethian, J.A.: Two-phase viscoelastic jetting. J. Comput. Phys. 220(2), 568-585 (2007)

11. Pillapakkam, S.B., Singh, P.: A level-set method for computing solutions to viscoelastic two-phase flow. J. Comput. Phys. 174(2), 552-578 (2001)

12. Goktekin, T.G., Bargteil, A.W., O’Brien, J.F.: Method for animating viscoelastic fluids. ACM Trans. Graph. 23(3), 463-468 (2004)

13. Losasso, F., Shinar, T., Selle, A., Fedkiw, R.: Multiple interacting fluids. In: SIGGRAPH 2006. ACM TOG 25, pp. 812-819 (2006)

14. Irving, G.: Methods for the physically based simulation of solids and fluids. Department of Computer Science, Stanford, Palo Alto (2007)

15. Chilcott, M.D., Rallison, J.M.: Creeping flow of dilute polymer solutions past cylinders and spheres. J. Non-Newton. Fluid Mech. 29, 381-432 (1988)

16. Singh, P., Leal, L.G.: Finite-element simulation of the start-up problem for a viscoelastic fluid in an eccentric rotating cylinder geometry using a third-order upwind scheme. Theor. Comput. Fluid Dyn. V5(2), 107-137 (1993)

17. Khismatullin, D., Renardy, Y., Renardy, M.: Development and implementation of VOF-PROST for 3D viscoelastic liquid-liquid simulations. J. Non-Newton. Fluid Mech. 140(1-3), 120-131 (2006)

18. Trebotich, D., Colella, P., Miller, G.H.: A stable and convergent scheme for viscoelastic flow in contraction channels. J. Comput. Phys. 205(1), 315-342 (2005)

19. Chang, Y., Hou, T., Merriman, B., Osher, S.: Eulerian capturing methods based on a level set formulation for incompressible fluid interfaces. J. Comput. Phys. 124, 449-464 (1996)

20. Sussman, M., Puckett, E.G.: A coupled level set and volume-of-fluid method for computing 3D and axisymmetric incompressible two-phase flows. J. Comput. Phys. 162(2), 301-337 (2000)

21. Tatebe, O.: The multigrid preconditioned conjugate gradient method. In: 6th Copper Mountain Conference on Multigrid Methods, Copper Mountain, Colorado, 1993

22. Briggs, W.L., Henson, V.E., McCormick, S.: A Multigrid Tutorial, 2nd edn. SIAM, Philadelphia (2000)

23. van Leer, B.: Towards the ultimate conservative difference scheme. V. A second-order sequel to Godunov's method. J. Comput. Phys. 100, 25-37 (1979)

24. Sussman, M.: A second order coupled level set and volume-of-fluid method for computing growth and collapse of vapor bubbles. J. Comput. Phys. 187(1), 110-136 (2003)

25. Sussman, M.: A parallelized, adaptive algorithm for multiphase flows in general geometries. Comput. Struct. 83, 435-444 (2005)

26. Hadamard, J.: Movement permanent lent d'une sphere liquide et visqueuse dans un liquide visqueux. C. R. Acad. Sci. Paris 152, 1735-1738 (1911)

27. Rybczynski, W.: Uber die fortschreitende Bewegung einer flussigen Kugel in einem zahen Medium. Bull. Int. Acad. Sci. Cracovia Cl. Sci. Math. Natur, 40-46 (1911)

28. Bhaga, D., Weber, M.E.: Bubbles in viscous liquids: shapes, wakes and velocities. J. Fluid Mech. Digit. Arch. 105(1), 61-85 (2006)

29. Bright, A.: Minimum drop volume in liquid jet breakup. Chem. Eng. Res. Des. 63, 59-66 (1985)

30. Richards, J.R., Lenhoff, A.M., Beris, A.N.: Dynamic breakup of liquid-liquid jets. Phys. Fluids 6, 26402655 (1994)

31. Hirt, C.W., Nichols, B.D.: Volume of fluid (VOF) method for the dynamics of free boundaries. J. Comput. Phys. 39(1), 201-225 (1981)

32. Brackbill, J.U., Kothe, D.B., Zemach, C.: A continuum method for modeling surface tension. J. Comput. Phys. 100(2), 335-354 (1992)

33. Johnson, J.R.E., Dettre, R.H.: The wettability of low-energy liquid surfaces. J. Colloid Interface Sci. 21(6), 610-622 (1966)

34. Noh, D.S., Kang, I.S., Leal, L.G.: Numerical solutions for the deformation of a bubble rising in dilute polymeric fluids. Phys. Fluids A 5, 1315 (1993) 